\title{
Correspondence
}

\begin{abstract}
We welcome letters to the Editor concerning articles which have recently been published. Such letters will be subject to the usual stages of selection and editing; where appropriate the authors of the original article will be offered the opportunity to reply.

Letters should normally be under 300 words in length, double-spaced throughout, signed by all authors and fully referenced. The edited version will be returned for approval before publication.
\end{abstract}

\section{Fractures involving splitting of the humeral head}

Sir,

We read with interest the article by Chesser et al ${ }^{1}$ entitled 'Fractures involving splitting of the humeral head' in the April 2001 issue. Although we welcome the highlighting of these uncommon and complex injuries, we take issue with the broad interpretation of the diagnostic criteria used in this paper. We believe that a number of fractures included in this series fall outwith the criteria for true 'head-splitting fractures' and are more consistent with impression fractures. The distinction is important because the pathology, prognosis and management are very different.

According to most authoritative texts, ${ }^{2-4}$ a head-splitting fracture is so defined by the gross disruption of the anatomical head, and thus the articular surface, of the proximal humerus. This deprives articular fragments of their vascular supply and results in a high incidence of subsequent avascular necrosis. We believe that the examples depicted are not true head-splitting fractures. Figure 1 shows the trough line or double shadow described by Cisternino et $\mathrm{al}^{5}$ which is correctly described in the text as representing an impaction fracture from a posterior dislocation. However, the caption ascribes this appearance to a "splitting fracture" and this is incorrect. We interpret Figures 2 and 5 as showing neglected posterior dislocations of the glenohumeral joint with associated large reverse Hill-Sachs lesions. Figure 3 is a CT image showing a fracture of the anatomical neck of the humerus, with a virtually intact articular surface.

The examples shown are therefore not obviously different from those presented in a previous paper describing the management of posterior fracture-dislocation of the shoulder.

True head-splitting fractures are by definition devastating injuries to the articular surface and are rarely reconstructable by the minimal internal fixation techniques described in this paper. ${ }^{2}$ It has been the experience of several authors that hemiarthroplasty, although bringing its own problems and limitations, remains the most appropriate solution to the management of true head-splitting fractures. ${ }^{7-9}$ The authors used minimal internal fixation for three out of eight fractures in their series. We would acknowledge that this was probably the most appropriate treatment for these relatively straightforward injuries. However, no firm conclusions can be drawn from such a small subgroup and extrapolation of the use of this technique to the treatment of the more complex headsplitting fracture is doubtful. Careful adherence to accepted classi-

(C2002 British Editorial Society of Bone and Joint Surgery 0301-620X/02/713676 \$2.00

J Bone Joint Surg [Br] 2002;84-B:1084-7. fication systems is essential if meaningful comparisons are to be drawn from reported results.

T. O. WHITE, BMedSci, AFRCS

C. M. ROBINSON, BMedSci, FRCS Ed (Orth)

Royal Infirmary of Edinburgh

Edinburgh, UK.

1. Chesser TJS, Langdon IJ, Ogilvie C, Sarangi PP, Clarke AM. Fractures involving splitting of the humeral head. J Bone Joint Surg [Br] 2001;83-B:423-6.

2. Bigliani LU. Fractures of the proximal humerus. In: Rockwood CA, Matsen FA, eds. The shoulder. Philadelphia: WB Saunders 1998;278-334.

3. Bigliani LU. Fractures of the proximal humerus. In: Rockwood CA, Green DP, Bucholz RW. Fractures in adults. Philadelphia: JB Lippincott and Co 1991:871-927.

4. Norris TR. Fractures of the proximal humerus and dislocations of the shoulder. In: Browner BD, Jupiter JB, Levine AM, Traften PG eds.Skeletal trauma. Philadelphia: WB Saunders Co 1992;1201-90.

5. Cisternino SJ, Rogers LF, Stufflebam BC, Kruglik GD. The trough sign: a radiographic sign of posterior shoulder dislocation. Am J Roentgenol 1978;130:951-4.

6. Stableforth PG, Sarangi PP. Posterior fracture-dislocation of the shoulder: a superior subacromial approach for open reduction. $J$ Bone Joint Surg [Br] 1992;72-B:579-84.

7. Kristiansen B. Treatment of displaced fractures of the proximal humerus: transcutaneous reduction and Hoffmann's external fixation. Injury 1989;20:195-9.

8. Ko JH, Yamamoto R. Surgical treatment of complex fractures of the proximal humerus. Clin Orthop 1996;327:225-237.

9. Moeckel BH, Dines DM, Warren RF, Altchek DW. Modular hemiarthroplasty for fractures of the proximal part of the humerus. $J$ Bone Joint Surg [Am] 1992;74-A:884-9.

\section{Authors' reply:}

Sir,

We thank Messers White and Robinson for their interest in our article. Terminology can be confusing and we specifically described "fractures involving splitting of the humeral head" and not "head-splitting fractures". As we have stated in previous correspondence relating to this article, Neer described the head-splitting fracture with the articular surface fragmented into many disconnected pieces. ${ }^{2}$ We describe fractures including splitting of the humeral head involving the articular surface and in seven of the eight cases reported that there was only one fracture line and in no case articular comminution. It is incorrect to describe these as "large reverse Hill-Sachs lesions" since a Hill-Sachs lesion is by definition a compression fracture with "a separate avulsed fragment from the humerus practically never present".

We agree that careful adherence to accepted classification systems is essential if meaningful comparisons are to be drawn, but rare injuries are often difficult to classify, especially into a system which is known to have a significant inter- and intraobserver error. ${ }^{4,5}$ For this reason it is important to describe the fracture, rather than to fit it into an existing classification system.

It is our recommendation that all fractures of the proximal humerus should undergo adequate radiological screening to identify the pattern of the fracture. In the cases which we describe, open reduction and internal fixation seemed preferable to replacement of the humeral head. Furthermore, minimal internal fixation techniques with anatomical reduction will not compromise later replacement of the joint. 
T. J. S. CHESSER, FRCS (Trauma \& Orth)

I. J. LANGDON, FRCS (Trauma \& Orth)

C. OGILVIE, MD

P. P. SARANGI, MD, FRCS

A. M. CLARKE, FRCS Orth

Bristol Royal Infirmary

Bristol, UK

1. Chesser TJS, Langdon IJ, Ogilvie C, Sarangi PP, Clarke AM. Correspondence. J Bone Joint Surg [Br] 2001;83-B:1209-10.

2. Neer CS. Displaced proximal humeral fractures. Part 1. Classification and evaluation. J Bone Joint Surg [Am] 1970;52-A:1077-89.

3. Hill HA, Sachs MD. The grooved defect of the humeral head. Radiology 1940;35:690-700.

4. Sidor ML, Zuckerman JD, Lyon T, et al. The Neer classification system for proximal humeral fractures: an assessment of interobserver and intraobserver reproducibility. $J$ Bone Joint Surg [Am] 1993;75-A:1745-50.

5. Siebenrock K, Gerber C. The reproducibility of classification of fractures of the proximal end of the humerus. J Bone Joint Surg [Am] 1993;75-A:1751-5.

\section{Simple treatment for torus fractures of the distal radius}

Sir,

We read with interest the article in the November 2001 issue by Davidson et al $^{1}$ entitled 'Simple treatment for torus fractures of the distal radius'. This is a simple but very relevant message with respect to identifying and treating a subgroup of paediatric fractures. We agree that these fractures are benign.

The authors stated that in their study the injuries were seen in the fracture clinic at a mean of $1.1 \pm 3$.6 days after the injury. This implies that the patients were seen between 2.5 days before the injury and 4.7 days after the injury!

They have also stated that one of the 201 fractures was actually not a torus fracture but a greenstick fracture. In a prospective trial with specific interest in torus fractures of the distal radius conducted at a paediatric hospital $0.5 \%$ of the fractures were "misdiagnosed". When this is translated to a general fracture clinic where the junior doctors generally treat these fractures, the percentage would in all probability be higher. In the present medicolegal situation this can be a potential for litigation. In the same vein, the authors stated that more patients from the 'splint group' did not attend the follow-up clinic. When patients do not attend the clinic they do so at their own risk and the health-care personnel cannot be held responsible for their actions. Finally, the authors have not mentioned how many patients in their 'splint group' actually asked for a further clinic appointment.

We entirely agree that torus fracture of the distal radius is a separate entity with little chance of any significant complication and also that there is a place for these fractures to be treated in a splint in the compliant patient with understanding parents.

\section{G. KUMAR, MS, FRCS}

V. KAMATH, MS

Wrightington Wigan \& Leigh NHS Trust

Lancashire, UK.

1. Davidson JS, Brown DJ, Barnes SN, Bruce CE. Simple treatment for torus fractures of the distal radius. J Bone Joint Surg $[\mathrm{Br}]$ 2001;83-B:1173-5.

\section{Author's reply:}

Sir,

We thank Messers Kumar and Kamath for their comments in regard to our article. In response to their first point we would like to thank them for highlighting the problem with using means and standard deviations with non-parametric data!

We agree with the comments regarding 'misdiagnosis' of the fracture. Our study is only applicable to simple torus fractures. It does not apply to greenstick fractures. It is precisely for this reason that a visit to the fracture clinic is necessary after injury to confirm the diagnosis. It is essential that any person treating these injures, in accordance with our protocol, can recognise the difference between the two patterns of fracture. We also suggest that if there is any doubt in diagnosis between the two, a more 'traditional' approach to treatment should be used.

For the duration of our study all patients in both groups were given a follow-up appointment at three weeks. No patients in either group required any further follow-up visit after this.

C. E. BRUCE

Alder Hey Children's Hospital

Liverpool, UK

\section{Pyomyositis of the iliacus muscle in a child}

Sir I read with interest the case report by Peckett et $\mathrm{al}^{1}$ in the January 2001 issue entitled 'Pyomyositis of the iliacus muscle in a child'.

Recently, a nine-year-old girl presented to us as a case of possible septic arthritis of the hip. With further investigations including MRI, the diagnosis proved to be pyomyositis. Ultrasound of the hip was negative and the blood parameters suggested acute infection. The positive blood cultures and appropriate systemic antibiotics gave resolution of the symptoms in five days.

As suggested by the reports of Peckett et $\mathrm{al}^{1}{ }^{1} \mathrm{Macnicol}^{2}$ and Papadopoulos et al, ${ }^{3}$ we endorse the use of MRI for the early definitive diagnosis of suspected infection around the hip or the pelvis. Pyomyositis appears to be increasing in prevalence in temperate climates. Orthopaedic surgeons are usually involved in the initial decision-making and ultimate care of these patients. The diagnosis is often delayed because of lack of familiarity and the paucity of symptoms on initial evaluation. Other primary diagnoses usually considered included muscle strain, synovitis, early Perthes' disease, thrombophlebitis and neoplasm thus contributing to the diagnostic delay. Pyomyositis of the obturator internus, ${ }^{3}$ adductor, ${ }^{4}$ and psoas 5 giving similar diagnostic confusion has also been reported.

MRI confirms whether the inflammatory process is localised to the muscle or is secondary to an infection in the bone. ${ }^{2}$

M. B. RAJESH, FRCS

Kent \& Canterbury Hospital

Canterbury, UK.

1. Peckett WRC, Butler-Manuel A, Apthorp LA. Pyomyositis of the iliacus muscle in a child. J Bone Joint Surg [Br] 2001;83-B:103-5.

2. Macnicol MF. Editorial. Patterns of musculoskeletal infection in childhood. J Bone Joint Surg [Br] 2001;83-B:1-2.

3. Papadopoulos M, Chugh S, Fitzgerald R, Thomas RJ. Obturator internus pyomyositis. Orthopaedics 2000;23:383-4.

4. De Boeck H, Noppen L, Desprechins B. Pyomyositis of the adductor muscles mimicking an infection of the hip: diagnosis by magnetic resonance imaging: a case report. J Bone Joint Surg [Am] 1994;76-A:747-50.

5. Perry J, Barrack RL, Burke SW, Haddad RJ. Psoas abscess mimicking a septic hip: diagnosis by computed tomography. J Bone Joint Surg [Am] 1985;67-A:1281-3.

\section{Authors' reply:}

Sir,

We were interested to learn that Mr Rajesh has had a similar case of pyomyositis around the hip in a child of the same age. He 
confirms the value of MRI in the investigation of such cases which allowed early diagnosis and resolution of the condition with appropriate systemic antibiotic treatment.

It may well be that pyomyositis is increasing in prevalence in temperate climates and we believe that it is important therefore that orthopaedic surgeons are aware of this differential diagnosis which we have shown can readily be diagnosed in its early stages by MRI.

We suspect that before the availability of MRI similar cases probably progressed to the formation of an abscess around the hip which required surgical drainage leading to increased morbidity. With greater clinical awareness of this condition and more readily available MRI pyomyositis will be detected at an early stage eliminating the need for surgical treatment.

W. R. C. PECKETT, FRCS

A. BUTLER-MANUEL, FRCS

L. A. APTHORP, FRCR

Conquest Hospital

St Leonards-on-Sea, UK.

\section{Treatment of isolated injuries of the lunotriquetral ligament}

\section{Sir,}

I read with interest the article in the September 2001 issue by Shin et al entitled 'Treatment of isolated injuries of the lunotriquetral ligament ${ }^{1}$ and wish to thank the authors for establishing that isolated lunotriquetral arthrodesis has an unacceptable rate of complications and an overall poor outcome.

The authors performed a power calculation to show that the size of their samples would allow the detection of a difference of 1.5 standard deviations between any two of the three groups. A power analysis is traditionally used in prospective studies to determine the size of the samples required to detect a predetermined effect, with a given $\beta$ probability, usually 0.8 . $^{2}$ To use such a calculation in reverse to determine the size of the difference for a given sample is unorthodox. A difference of 1.5 standard deviations between groups is a very large effect, using the standard Cohen ratios of $0.2,0.5$ and 0.8 standard deviations to indicate small, medium and large effects respectively. ${ }^{3}$ These figures would indicate that this study is underpowered, leading to potentially significant results being dismissed.

For example, the authors state that the DASH score for arthrodesis was worse than that for the other groups, but that it was not statistically significant. This is probably because the number of patients studied was too small. It is also stated that the three treatment groups were statistically similar for a number of factors including the mechanism of injury, the grip strength and the range of movement, etc. This again could have been due to the lack of power of the study and not the inherent similarity of the groups.

The main difference between the groups is the length of followup available. The mean follow-up was $6.7,10.5$ and 16.6 years for the repair, arthrodesis and reconstruction groups, respectively. This implies that the standard treatment for this injury changed over time and in effect the authors are using historical controls. This is of course a valid means of study, but not one that is clearly stated in the methods.

The conclusion was that ligament repair or reconstruction should be the operation of choice for this type of injury. That may be the case, but as with most retrospective studies, a properly controlled, adequately powered study, with non-operative management as one of the groups, is required to demonstrate this rigorously.

I. WRIGHT, FRCS

Royal Shrewsbury Hospital

Shrewsbury, UK.
1. Shin AY, Weinstein LP, Berger RA, Bishop AT. Treatment of isolated injuries of the lunotriquetral ligament: a comparison of arthrodesis, ligament reconstruction and ligament repair. J Bone Joint Surg [Br] 2001;83-B:1023-8.

2. Freedman KB, Back S, Bernstein J. Sample size and statistical power of randomised, controlled trials in orthopaedics. J Bone Joint Surg [Br] 2001;83-B:397-402.

3. Cohen J. Statistical power analysis for the behavioural sciences. Second ed. Hillsdale, NJ: Lawrence Erlbaum Associates, Inc, 1988.

\section{Authors' reply:}

Sir,

We thank Mr Wright for his letter. In a retrospective study, the use of a power analysis is quite appropriate since the size of the sample is predetermined by the number of records available for review. A power calculation helps to define the threshold at which differences between groups are not likely to be detected because of sample size. Furthermore, dismissal of all of our results on the basis of low statistical power is not justified. There was sufficient power to detect several factors which significantly affected the outcome. Mr Wright is correct in recognising that other variables in which the observed differences were less than 1.5 standard deviations may be significant with larger clinical numbers, a typeII error.

Another concern expressed was variation in group characteristics. We reported that the three treatment groups (arthrodesis, ligament reconstruction and ligament repair) were statistically similar with respect to age, hand dominance, the mechanism of injury, chronicity, workers' compensation status, the pretreatment range of movement and grip strength. In our paper, preoperative differences in range of movement and grip strength of the wrist between groups were evaluated using analysis of covariance. This served to minimise any potential effect as a result of possible preoperative differences in these variables.

Although withholding treatment for an identified medical problem may be required to provide complete assurance of the results of intervention, our study is not unique in lacking this control group. This is a recognised problem with retrospective reviews in general. Ultimately, it was our desire to provide the most thorough analysis possible given the limitations of a retrospective study. Our conclusions are based on these results, without speculation or personal bias. The factors identified as significant are valid and important for clinicians to consider in practice. Future prospective studies may allow the identification of other significant variables.

A. T. BISHOP, MD

A. Y. SHIN, MD

Mayo Clinic

Rochester, USA.

\section{Prevention of pulmonary embolism by a foot sole pump}

Sir,

We write with reference to the article by Asano et $\mathrm{al}^{1}$ in the November 2001 issue entitled 'Prevention of pulmonary embolism by a foot sole pump'. I have some concerns with regard to the methodology of the study.

First, pulmonary embolism was defined as a new defect detected by pulmonary perfusion scintigraphy which was performed before and at one week after the operation with comparison of the two recordings. It is recognised that fat and bone-marrow embolic events occur during hip arthroplasty, 
especially associated with the preparation of the femur and the insertion of the femoral component. ${ }^{2,3}$ Such embolic events could result in defects detected by perfusion scintigraphy, and their occurrence would not have been influenced by the use of foot pumps. It might have been more appropriate if the baseline reading had been performed early in the postoperative period rather than before hip surgery.

Secondly, the authors rightly acknowledged that perfusion defects may occur as a result of local hypoventilation. It is common practice in assessing pulmonary embolism to perform combined ventilation/perfusion scans to distinguish matched from unmatched defects. This was not performed in this study nor was there any mention of the incidence of postoperative respiratory problems in the control and treatment groups.

\section{CHARALAMBOUS, MRCS}

D. ELLIS, FRCS Orth

Manchester Royal Infirmary,

Manchester, UK.

1. Asano H, Matsubara M, Suzuki K, Monta S, Shinomiya K. Prevention of pulmonary embolism by a foot sole pump. J Bone Joint Surg [Br] 2001;83-B:1130-2.

2. Koessler MJ, Pitto RP. Fat and bone marrow embolism in total hip arthroplasty. Acta Orthop Belg 2001;67:97-109.

3. Pitto RP, Blunk J, Kossler M. Transesophageal echocardiography and clinical features of fat embolism during cemented total hip arthroplasty: a randomised study in patients with a femoral neck fracture. Arch Orthop Trauma Surg 2000;120:53-8.

\section{Author's reply:}

Sir

We thank Mr Charalambous for his comments and we agree that fat and bone-marrow embolic events occur during hip arthroplasty and that our defects may include them. However, we disagree that a baseline reading should have been performed early in the postoperative period for three reasons. First, it is difficult to perform scintigraphy just after surgery when the condition of the patient is not stable. Secondly, deep-vein thrombosis begins during surgery and a new defect early in the postoperative period does not necessarily mean that it is the result of a fat and marrow embolic event. Finally, we wished to know the usefulness of a foot pump for the reduction of pulmonary embolism as a whole.

Perfusion defects may occur as a result of local hypoventilation. It is possible that if we had included such cases that the incidence of pulmonary embolism would have been lower than we showed. However, a foot pump cannot prevent local hypoventilation and a reduction of the incidence of new defects means that a pulmonary embolism has been prevented. The fact that we did not use ventilation scans does not deny the usefulness of a foot pump. In addition, there were no respiratory problems among the control and treatment groups, and there were no abnormal signs on radiographs of the chest.

H. ASANO, MD

Tokyo Medical and Dental University

Tokyo, Japan. 